\title{
Cuestionário de Perdón en Divorcio-Separación (CPD-s): adaptação e evidências de validade
}

\author{
Questionnaire on Forgiveness in Divorce-Separation: \\ fidaptation and Euidence of Ualidity \\ Cuestionario de Perdón en Divorcio-Separación (CPD-S]: \\ adaptación y evidencias de validez
}

\author{
Ricardo Neves Couto, Patrícia Nunes da Fonsêca* \\ Universidade Federal da Paraiba \\ Emerson Diógenes de Medeiros \\ Universidade Federal do Piauí - UFPI
}

Doi: http://dx.doi.org/10.12804/revistas.urosario.edu.co/apl/a.5026

\section{Resumo}

Por meio de dois estudos, objetivou-se adaptar o Cuestionário de Perdón en Divorcio-Separación (CPD-S) para o contexto brasileiro, fornecendo evidências de validade fatorial e consistência interna. No Estudo 1 participaram 204 pessoas divorciadas com idade média de 41,98 ( $D P=11,99), 74 \%$ do sexo feminino, que responderam ao CPD-S e a perguntas sociodemográficas. A análise fatorial exploratória revelou uma estrutura unifatorial, explicando $55,92 \%$ da variância total e alfa de Cronbach de 0,79. No Estudo 2 participaram 200 pessoas divorciadas distribuídas equitativamente entre os sexos e com idade média de 38,72 anos ( $D P=$ 11,51 ), que responderam os mesmos instrumentos do estudo anterior. Os resultados da análise fatorial confirmatória corroboraram a estrutura unifatorial, com alfa de Cronbach de 0,73. Conclui-se que os resultados demonstram evidências quanto a validade fatorial e consistência interna do instrumento, além de mensurar o construto de forma similar independente do gênero ou status atual de relacionamento dos participantes. Palavras-chave: perdão, divórcio, testes psicológicos, relacionamento, validade.

\section{Abstract}

\begin{abstract}
Through two studies, we aimed to adapt the Questionnaire on Forgiveness in Divorce-Separation (Cuestionário de Perdón en Divorcio-Separación - CPD-S) to the Brazilian context, providing evidence of factorial validity and internal consistency. In study 1, participants were 204 divorced individuals with a mean age of 41.98 (SD $=11.99), 74 \%$ women, who answered CPD-S and demographic questions. The exploratory factor analysis showed a one-factor structure, explaining $55.92 \%$ of the total variance, and a Cronbach's alpha of .79. In study 2 , participants were 200 divorced individuals equally
\end{abstract}

* Correspondência. Correio eletrônico: patynfonseca@hotmail.com

Cómo citar este artículo: Couto, R. N., Da Fonsêca, P. N., \& De Medeiros, E. D. (2018). Cuestionário de Perdón en DivorcioSeparación (CPD-S): adaptação e evidências de validade. Avances en Psicología Latinoamericana, 36(1), 183-196. doi: http:// dx.doi.org/10.12804/revistas.urosario.edu.co/ap1/a.5026 
distributed across gender with a mean age of 38.72 (SD $=11.51$ ), answering the same instruments as in study 1 . Confirmatory factor analysis results corroborated the one-factor structure, with a Cronbach's alpha of .73. Furthermore, the factorial invariance across gender and current relationship status was tested. It can be concluded that the results show evidence of factorial validity and internal consistency in addition to measuring the construct across gender and current relationship status. Keywords: Forgiveness, divorce, psychological tests, relationship, validity.

\section{Resumen}

Por medio de dos estudios, se tuvo como objetivo adaptar el Cuestionario de Perdón en Divorcio-Separación (CPD-S) para el contexto brasilero, proporcionando evidencias de validez factorial y consistencia interna. En el estudio 1 participaron 204 personas divorciadas con edad promedio de 41,98 (DP $=11,99), 74 \%$ de sexo femenino, que respondieron al CPD-S y a preguntas sociodemográficas. El análisis factorial exploratorio reveló una estructura unifactorial, explicando 55,92\% de varianza total y alfa de Cronbach de 0,79 . En el estudio 2 participaron 200 personas divorciadas distribuidas equitativamente entre los sexos y con edad promedio de 38,72 años ( $D P=11,51$ ), que respondieron los mismos instrumentos del estudio anterior. Los resultados del análisis factorial confirmatorio corroboraron la estructura unifactorial, con alfa de Cronbach de 0,73 . Se concluye que los resultados demuestran evidencias en cuanto a la validez factorial y consistencia interna del instrumento, además de medir el constructo de forma similar independiente del género o estatus actual de relación de los participantes.

Palabras clave: perdón, divorcio, tests psicológicos, relación, validez.

\section{Introdução}

As pessoas, em sua maioria, casam-se com a ideia de que o matrimônio é para a vida toda.
Todavia, nem sempre é o que acontece, há momentos em que os cônjuges decidem por fim ao casamento, sendo o divórcio a última decisão tomada por cônjuges que reconhecem não terem superado momentos de dificuldades após inúmeras tentativas de resolução de conflitos no casamento (Alves, Cúnico, Arpini, Smaniotto \& Bopp, 2014).

$\mathrm{O}$ ato de conceder o perdão ao ex-cônjuge pela incapacidade de manter a união, destruir planos, sonhos e não atingir as expectativas do relacionamento está presente no processo de desintegração do casal. Contribuindo positivamente com o inevitável convívio entre os ex-cônjuges, sobretudo naquelas famílias com filhos, em que a estrutura familiar altera-se, porém a organização parental é mantida (Yárnoz-Yaben, Garmendia \& Comino, 2015).

Nessa direção, o convívio continua nas situações que desencadeiam conflitos de interesses e múltiplas batalhas (e.g. financeiras, guardas dos filhos). Além disso, tudo que foi vivenciado requer mudanças e para isto, há necessidade de se manejar a mágoa, raiva, culpa, vergonha e perda em si mesmo (Alves et al., 2014). Por consequente, pessoas divorciadas experienciam um acentuado grau de extrema ansiedade (distress) psicofisiológico, perdas acentuadas na segurança econômica e no suporte social, alterações depreciativas na percepção do self e desestabilização emocional nos sistemas de procura e prestação de cuidados (Lamela, Figueredo, Bastos \& Martins, 2014).

Observa-se, pois, ser necessário seguir avançando em uma investigação sobre os impactos dos divórcios, identificando variáveis que possam servir para intervenções positivas e preventivas (Yárnoz-Yaben \& González, 2012), que de alguma maneira possam melhorar o bem-estar dos envolvidos. Destacando o manejo por meio da vontade de perdoar, por promover o bem-estar físico e psicológico (Leime, Luna, Leite \& Neto, 2012) e ser ao nível pessoal, comunitário e familiar, determinante na qualidade das relações das pessoas (Neto \& Mullet, 2012). Portanto, considerando o 
perdão como fator de manutenção do bom funcionamento físico e mental, segue-se com detalhes da temática, a seguir.

\section{Perdão}

É recente o interesse das ciências humanas e da saúde em estudar o perdão. Iniciando na comunidade terapêutica depois da publicação do livro "Forgive and Forget: healing the hurts we don't deserve”, de Lewis Smedes, em 1984 (Blocher \& Wade, 2010). Contudo, comumente é relacionado ao Judaísmo, Cristianismo e Islamismo, propagando a ideia de que as pessoas, por serem perdoadas por Deus, deveriam perdoar seus pares e transgressores. Entretanto, o perdão não deve ser visto estritamente como uma virtude religiosa, sendo essa proximidade apontada como a possível causa de desinteresse pelo construto por parte dos pesquisadores (McCullough \& Witvliet, 2002).

Etimologicamente a palavra perdão provém do latim, verbo perdonare (inicialmente com os significados de dar e conceder) e o substantivo perdonum, composto do prefixo per (agrega os sentidos de por através de, e de plenitude), que pode significar perfeição da ação e também afastamento, mais o substantivo donum (dom). Assim, perdoar significaria entregar um dom completamente sem exigir nada em troca (Beltrão, 2012). No tocante à abordagens mais psicológicas, a falta de uma definição consensual é encarada como um dos problemas mais importantes do campo (Enright \& Coyle, 1998; Enright, Freedman \& Rique, 1998; Worthington, 2005), sendo alcançado primeiramente o acordo sobre o que não pode ser caracterizado como perdão.

Isso posto, Enright e Coyle (1998) destacam as distinções feitas de construtos que frequentemente são, erroneamente, usados indistintamente ao ato de perdoar: (i) Absolvição, conceito relacionado à Justiça, implica perdoar legalmente alguém da pena consequente à transgressão cometida; (ii) Desculpas, finge-se que não está ofendido e conclui que não houve intenção; (iii) Esquecimento, sugere a ideia de que a memória da ofensa foi apenas suprimida da consciência; (iv) Negação, refere-se, unicamente, à uma indisposição ou incapacidade para perceber que uma afronta tenha ocorrido; (v) Reconciliação, indica a restauração das relações, porém pode-se perdoar sem reconciliar-se. Não obstante, evidencia-se a importância de conhecer as produções e pontos de convergência na literatura consultada.

É possível apontar Robert Enright e o Grupo de Estudos sobre Desenvolvimento Humano como pioneiros nos estudos sobre a inclinação para pedir perdão e a capacidade de perdoar ao longo do ciclo de vida (Enright \& The Human Development Study Group, 1991). Para os que compõem este grupo, o perdão é definido como "uma atitude moral e vontade de abdicar do direito ao ressentimento, julgamentos e comportamentos negativos em direção a quem ofendeu injustamente, nutrindo simultaneamente sentimentos desmerecidos de compaixão, misericórdia e, até mesmo, de amor ao ofensor" (Enright, Freedman \& Rique, 1998, pp. 46-47).

Por sua vez, Worthington (2005) menciona que existem dois tipos de formação do perdão. $\mathrm{O}$ decisional, que envolve mudança nas intenções de se comportar em relação ao transgressor, e o emocional, que caracteriza-se pela substituição de emoções negativas por outras de cunho positivo. Esta visão foi influenciada por DiBlasio (1998), que propôs a ideia de perdão baseado em decisão, definindo-o como uma mudança na força de vontade empregada pela pessoa ofendida com a finalidade de suprimir comportamentos nocivos direcionados ao ofensor.

Nesta oportunidade, McCullough e Witvliet (2002) são eleitos os estudiosos que de forma mais simples e direta operacionalizam o construto. Estes autores partilham da ênfase na conexão emoção-motivação de Worthington, e asseveram que o perdão ocorre quando há mudanças nas motivações que envolvem a cessação de vingança 
e um decréscimo da fuga. Logo, caracteriza-se como um conjunto de mudanças intraindividuais e pró-sociais que a vítima experimenta quando perdoa a um ofensor dentro de um contexto específico (McCullough, 2000), sendo o ato de perdoar independente da ação de reconciliar.

Portanto, entende-se o perdão como uma construção social e psicológica, na qual a pessoa tem consciência de ter sido vítima de uma injustiça, compreende que o ofensor não merece compaixão e amor, podendo conceder o perdão ou não, mas abandona os sentimentos negativos e oferece-lhe sentimentos positivos. Dessa forma, ao proporcionar uma abdicação do sentimento de vingança em situações de transgressões, o perdão configura-se como promotor de harmonia social (Gouveia et al., 2015).

Não obstante, verificam-se convergências nas diferentes perspectivas acerca do perdão (Strelan \& Covic, 2006), a saber: (i) o perdão implica uma diminuição no ressentimento; (ii) reconhecimento de uma ofensa injusta, dando o direito de estar ferido e; (iii) a desistência da raiva e da vingança, nutrindo sentimentos positivos ao transgressor. Considerando, assim, o perdão um objeto de investigação fundamental para a psicologia e suas diferentes áreas (Leime et al., 2012).

\section{Perdão no âmbito do divórcio}

No âmbito do divórcio, o perdão integra-se em uma narrativa coerente, dirimindo as fantasias de vingança, ódio e ressentimento, proporcionando que essa energia seja canalizada para novos projetos (Yárnoz-Yaben et al., 2015), superação e habilidades de estabelecer novos relacionamentos (Toussaint et al., 2012). Atribuindo menores níveis de culpa e um julgamento mais brando, com menos conflitos e problemas comportamentais relacionados ao ex-parceiro(a) (Yárnoz-Yaben \& González, 2012).

Um número crescente de publicações na área específica da ruptura do vínculo aborda o perdão com diferentes aspectos de adaptação à nova situação, sinalizando que níveis mais elevados de perdão estão associados a uma melhor adaptação ao divórcio. Destacam-se as investigações acerca do ajuste das crianças ao divórcio dos pais (Freedman \& Knupp, 2003), modelos de enfrentamento e relação com o ex-parceiro (Mazor, Batiste-Harel \& Gampel, 1998) e saúde mental (Rye, Folck, Heim, Olszewski \& Traina, 2004)

Yárnoz-Yaben, Comino e Garmendia (2012) encontraram aspectos da adaptação ao divórcio relacionados ao grupo familiar quando o ex-cônjuge é perdoado. Pais divorciados que perdoam seus ex-parceiros são capazes de realizar um reenquadramento cognitivo das ofensas passadas (Bonach \& Sales, 2002), diminuindo também os problemas comportamentais quando relacionado com o apoio recebido do ex-parceiro (Yárnoz-Yaben et al., 2012). Logo, reconhecendo a importância do perdão na manutenção das relações sociais e promoção de bem-estar subjetivo, justifica-se o uso de instrumentos psicométricos como eficazes ferramentas no êxito da sua mensuração. Dessa forma, segue-se com a descrição de alguns dos principais instrumentos disponíveis na literatura.

\section{Mensuração do Perdão}

Atualmente, no cenário internacional, realizando um levantamento de dados do Periódico CAPES e Google Acadêmico no dia 19 de maio de 2016, utilizando "escala" e "perdão" como descritores, foram encontradas diferentes escalas que permitem avaliar o perdão.

A Wade's Scale Forgiveness, tomando o perdão como estado, momentâneo e passageiro, foi desenvolvida por Wade (1989) com 83 itens focalizando uma definição operacional de perdão. Baseando-se em duas escalas desse instrumento supracitado, evasão e vingança, McCullough et al. (1998) desenvolveram o Transgression Related Interpersonal Motivations Inventory (TRIM), preenchendo a lacuna de instrumentos que avaliam 
as mudanças motivacionais. Atualmente é composto por 18 itens e três escalas (benevolência, evitamento e vingança), com um alfa de Cronbach geral de 0,93 (McCullough, Root \& Cohen, 2006).

Buscando suprir a necessidade de uma escala relativamente breve contendo perguntas que contemplem respostas positivas e negativas em relação aos infratores, Rye et al. (2001) desenvolveram a Forgiveness Scale (Escala de Perdão), a qual se estrutura em duas dimensões: presença de aspectos positivos (5 itens) e ausência de negativos (10 itens), com alfas de Cronbach de 0,85 e 0,86, respectivamente.

Quanto às medidas que avaliam a disposição para perdoar, entendida como uma tendência geral, estável ao longo do tempo e em diferentes situações, destaca-se a Willingness to Forgive (WTF). Construída por DeShea (2003), é composta por 12 itens em forma de cenários, nos quais os participantes indicam a disposição para perdoar a pessoa destacada em cada cenário. Adaptada para o Brasil por Gouveia et al. (2015), teve sua estrutura unifatorial comprovada, $\mathrm{x}^{2} / \mathrm{g} .1$. $=3,39$, $\mathrm{GFI}=0,90, \mathrm{AGFI}=0,85, \mathrm{CFI}=0,87$ e RMSEA $=$ 0,089 (IC90\% = 0,075-0,104), além de apresentar evidencias de fidedignidade (alfa de Cronbach $=$ $0,85)$ e invariância fatorial adequadas.

Objetivando avaliar atitudes em relação ao perdão, o Enright Forgiveness Inventory (EFI), criado por Subkoviak et al. (1995), baseia-se no modelo teórico das atitudes proposto por Enright et al. (1991) e apresenta itens relativos às seis dimensões teóricas do perdão (afetos positivos e negativos, julgamentos positivos e negativos, comportamentos positivos e negativos). Teve suas qualidades psicométricas avaliadas no Brasil por Rique, Camino, Santos e Gouveia (2009) resultando na Escala de Atitudes para o Perdão, composta por 60 itens e seis subescalas, apresenta indicadores de ajustes adequados $[\mathrm{CFI}=0,94$; TLI $=0,94$; RMSEA $=0,057(0,055-0,060)]$.
Por fim, buscou-se conhecer os instrumentos disponíveis para avaliar o perdão em situações específicas, não hipotéticas, como é o caso do divórcio. Para tanto, realizou-se busca na base de dados Dialnet, no dia 19 de maio de 2016, utilizando "Evaluation of Forgiveness in Divorce" como descritores, tendo como resultado apenas um artigo. Esse apresentou um instrumento espanhol, composto por 5 itens, com objetivo de avaliar o perdão frente ao ex-parceiro (a): o Cuestionário de Perdón en Divorcio-Separación (CPD-S, Yárnoz-Yaben \& González, 2012). Ancorando-se na perspectiva teórica do perdão interpessoal (Enright et al., 1998), apresenta a vantagem de abordar aspectos do perdão, tais como não guardar ressentimentos, rancor e raiva, diminuição de sentimentos negativos e aumento de compaixão frente ao ex-cônjuge.

$\mathrm{O}$ CPD-S, inicialmente proposto com 15 itens, sofreu modificações sugeridas por pesquisadores especialistas no divórcio e teve suas qualidades psicométricas verificadas em uma amostra preliminar de divorciados. A versão final com 5 itens e um único fator, explicou $48 \%$ da variância total (Yárnoz-Yaben \& González, 2012), com precisão (alfa de Cronbach $=0,77$ ) e validade de construto igualmente adequada. Nos últimos anos, o CPD-S é utilizado com o fim de analisar o lugar do perdão em situações de divórcio e sua relação com a adaptação, o bem-estar subjetivo e satisfação com a vida (Yárnoz-Yaben et al., 2015).

As qualidades psicométricas e vantagens apresentadas pelo CPD-S parecem justificar o esforço empreendido neste estudo em preencher a lacuna de instrumentos disponíveis no Brasil que mensurem o perdão no âmbito do divórcio. Dessa forma, busca-se contribuir com a produção científica, tida como recente, da temática. Pelo exposto, o presente estudo tem como objetivo principal apresentar a adaptação para o português brasileiro e verificar evidências de validade fatorial e consistência interna do Cuestionário de Perdón en Divorcio-Separación (CPD-S) em duas amostras independentes 
brasileiras. Para tanto, foram realizados dois estudos empíricos, apresentados a seguir.

\section{Método}

Estudo 1. Evidências preliminares de validade e precisão da CPD-S.

\section{Participantes}

Contou-se com uma amostra por conveniência (não-probabilista) composta por 204 indivíduos, apresentando como critério de inclusão ter passado por um processo de divórcio (idades de 18 a 84 anos, $M=41,98, D P=11,99)$. A maioria do sexo feminino (74\%), oriundos de 10 estados brasileiros das regiões Nordeste, Sudeste e Sul, em sua maioria do Piauí (44,12\%) e Paraíba (17,33 \%). A duração do relacionamento que ocasionou em divórcio era em média 10,51 anos $(D P=7,47)$, além disso, os participantes afirmaram estar divorciados em média á 8,55 anos $(D P=8,14)$ e a maioria $(59,3 \%)$ não está em outro relacionamento amoroso. Quanto à escolaridade, a maioria $(27,5 \%)$ possui apenas o ensino médio completo. A renda familiar modal foi a categoria “entre R \$789 e R \$1.576" (22,1\%), seguida de “entre R\$2.365 e R\$4.728” (21,6\%).

\section{Instrumentos}

Os participantes responderam a um livreto contendo os seguintes instrumentos:

Cuestionario de Perdón en Divorcio-Separación (CPD-S): criado em língua espanhola, objetiva avaliar o perdão frente ao ex-parceiro (Yárnoz-Yaben \& González, 2012). Os 5 itens pertencem a um único fator (e.g. Eu perdoei o meu/minha ex-parceiro(a); Espero que meu/minha ex-parceiro(a) receba o que merece por todo mal que elelela me $f e z$ ) e são avaliados em uma escala Likert de cinco pontos $(1=$ discordo totalmente e 5 = concordo totalmente). Validado originalmente com uma amostra de 223 pais divorciados espanhóis, apresenta boa consistência interna (alfa de Cronbach $=0,77)$ e uma única dimensão $(48 \%$ da variância explicada).

Questionário Sociodemográfico: conjunto de perguntas que objetivaram caracterizar os participantes. Consta de questões sobre idade, sexo, renda, tempo ao lado do ex-cônjuge, tempo de divórcio e status atual de relacionamento amoroso.

\section{Procedimento}

Inicialmente, foi realizada a tradução do CPD-S por meio da técnica de backtranslation (Pasquali, 2010), auxiliada por profissionais bilíngues (Português-Espanhol). Assim, a medida foi traduzida por psicólogos bilíngues e retraduzida para o espanhol, com a colaboração de duas pessoas bilíngues independentes, que não conheciam o instrumento (tradução às cegas). Após comparar as versões e suas equivalências, atentos às diferenças culturais de cada idioma, foi verificado que a versão em português refletia a versão espanhola.

Em seguida, procedeu-se a validação semântica, seguindo os procedimentos estabelecidos por Pasquali (2010). Para isto, contou-se com 10 participantes da população alvo, distribuídos equitativamente entre homens e mulheres. Na ocasião, verificou-se não haver dificuldades de leitura e/ou interpretação dos itens, o que permitiu prosseguir com o uso do instrumento.

Para a aplicação dos instrumentos, três colaboradores, devidamente treinados previamente, visitavam residências, escolhidas acidentalmente em estados brasileiros. Neste momento eram informados o objetivo geral do estudo, o caráter voluntário da participação, bem como garantido o anonimato das respostas e a possibilidade de desistir de sua participação no estudo a qualquer momento, sem ônus. As coletas também foram feitas online por meio da plataforma Google Docs. Os indivíduos acessaram um endereço eletrônico que esteve disponível entre o período de maio de 2015 e abril de 2016, sendo contatados via e-mail, 
redes sociais e pessoalmente, permanecendo na amostra final apenas as pessoas que declararam já ter enfrentado um processo de divórcio. Estes participantes atestaram o seu consentimento através do Termo de Consentimento Livre e Esclarecido (TCLE) antes de responder individualmente aos instrumentos, que eram necessários, em média, 10 minutos para sua conclusão.

Destaca-se que foram asseguradas todas as recomendações éticas relacionadas às pesquisas com seres humanos, conforme o disposto na resolução 466/2012 do Conselho Nacional de Saúde, sendo a realização do estudo aprovado por um Comitê de Ética da Universidade Federal da Paraíba através da Plataforma Brasil (CAAE: 46788315.1.0000.5188; parecer $\left.n^{\circ} 1.197 .954\right)$.

\section{Análises de dados}

Com o software Factor 9,2 (Lorenzo-Seva \& Ferrando, 2013) investigou-se a dimensionalidade do CPD-S com o método Hull Comparative Fit Index (CFI; Lorenzo-Seva, Timmerman \& Kiers, 2011), a partir de uma análise fatorial exploratória categórica Unweighted Least Squares (ULS) com correlações policóricas. De acordo com o estudo de simulação de Lorenzo-Seva et al. (2011), o método Hull é um dos melhores disponíveis para estimar a dimensionalidade de um conjunto de itens. Além disso, pôde-se checar a consistência interna do alfa de Cronbach com base nas correlações policóricas. Estas análises foram conduzidas considerando o fato de que a escala Likert utilizada é composta de categorias ordenadas, não sendo possível considerá-la como contínua (Holgado-Tello, Chacón-Moscoso, Barbero-García \& Vila-Abad, 2010; Lara, 2014).

Com o software R (R Core Team, 2015) foram realizadas análises descritivas, que objetivou descrever os participantes, e o pacote ltm (Rizopoulos, 2006) foi utilizado para calcular, por meio do modelo de resposta graduada (Samejima, 1969), considerando a natureza ordinal da medida, o parâmetro discriminação dos itens (a).

\section{Resultados}

A matriz de correlações policóricas mostrou-se adequada para a análise fatorial exploratória ordinal ULS, tendo os índices Kaiser-Meyer-Olkin $(\mathrm{KMO})=0,73$ e o teste de esfericidade de Bartlett $=343,10(10) ; p<0,001$. A análise de dimensionalidade pelo método Hull (CFI) sugeriu uma solução unidimensional, resultando em um índice de ajuste Global Fit Index (GFI) =0,99. O fator retido (autovalor $=2,79$ ) explicou $55,92 \%$ da variância total dos itens. As cargas fatoriais dos itens do CPD-S e a consistência interna da escala são apresentadas na tabela 1 .

Tabela 1

Estrutura fatorial do Cuestionario de Perdón en Divorcio-Separación.

\begin{tabular}{|c|c|c|c|}
\hline Itens & A & Fator & $\mathrm{h}^{2}$ \\
\hline $\begin{array}{l}\text { 02. Não posso evitar de culpar meu/ } \\
\text { minha ex-parceiro(a) } \\
\text { por ter causado o fim do nosso rela- } \\
\text { cionamento. (R) }\end{array}$ & 0,50 & $0,45^{*}$ & 0,20 \\
\hline $\begin{array}{l}\text { 01. Estou com raiva do } \mathrm{meu} / \mathrm{minh} \\
\text { ex-parceiro(a). (R) }\end{array}$ & 0,78 & $0,61 *$ & 0,38 \\
\hline $\begin{array}{l}\text { 05. Espero que meu/minha ex-par- } \\
\text { ceiro(a) receba o que merece por } \\
\text { todo mal que ele/ela me fez. }(\mathrm{R})\end{array}$ & 0,88 & $0,66^{*}$ & 0,44 \\
\hline $\begin{array}{l}\text { 03. Eu perdoei o meu/minha } \\
\text { ex-parceiro(a). }\end{array}$ & 1,33 & $0,80^{*}$ & 0,64 \\
\hline $\begin{array}{l}\text { 04. Apesar do comportamento do } \\
\text { meu/minha ex-parceiro(a) ter me } \\
\text { machucado, não guardo rancor } \\
\text { dele(a). }\end{array}$ & 1,37 & $0,81 *$ & 0,65 \\
\hline Quantidade de itens & & 5 & \\
\hline Variância explicada (\%) & & 55,92 & \\
\hline Valor próprio & & 2,79 & \\
\hline$\Omega$ de McDonald & & 0,80 & \\
\hline$\alpha$ de Cronbach & & 0,79 & \\
\hline
\end{tabular}

Nota: a = parâmetro de discriminação (modelo de resposta graduada); * carga fatorial considerada satisfatória, isto é, $>|0,30|$. (R) $=$ Itens revertidos. $\mathrm{h}^{2}=$ comunalidade. $\alpha=$ alfa de Cronbach com base em correlações policóricas. 
Em resumo, os resultados apontam a pertinência da solução unifatorial, sugerindo evidências satisfatórias acerca da validade de construto do CPD-S. Todos os cinco itens tiveram saturações superiores a 0,30 no fator "Perdão no âmbito do divórcio", variando de 0,45 [item 02 , "Não posso evitar de culpar meu/minha ex-parceiro(a) por ter causado o fim do nosso relacionamento"'] a 0,81 [item 04, "Apesar do comportamento do $\mathrm{meu} / \mathrm{min}$ ha ex-parceiro(a) ter me machucado, não guardo rancor dele(a).'”. Ademais, o fator denominado de Perdão no âmbito do divórcio, apresentou alfa de Cronbach com base em correlações policóricas de 0,79 e ômega de McDonald de 0,80. Portanto, é possível observar que sua consistência interna pareceu igualmente satisfatória.

Vencida esta etapa, faz-se necessário averiguar a estrutura fatorial encontrada nesta ocasião, utilizando técnicas confirmatórias, a exemplo da Análise Fatorial Confirmatória (AFC) e invariância entre sexo e estar ou não em um novo relacionamento. Estes são objetivos do segundo estudo, que será descrito a seguir.

Estudo 2. Comprovação da estrutura fatorial e invariância do CPD-S.

\section{Participantes}

Contou-se com uma amostra acidental (não-probabilista) composta por 200 indivíduos, apresentando como critério de inclusão ter passado por um processo de divórcio (idades de 19 a 71 anos, $M=38,42, D P=11,51)$. Oriundos de 10 estados brasileiros das regiões Nordeste, Sudeste e Sul, em sua maioria da Paraíba $(29,32 \%)$ e do Piauí $(27,21 \%)$, foram distribuídos equitativamente entre os sexos (100 homens e 100 mulheres).

A duração do relacionamento que ocasionou em divórcio era em média 9,14 anos $(D P=6,29)$. Além disso, os participantes afirmaram estar divorciados em média a 5,84 anos $(D P=6,01), 51,5 \%$ não estão atualmente em outro relacionamento amoroso e possui pós-graduação (31\%). A renda familiar modal foi a categoria "entre $\mathrm{R} \$ 1.577 \mathrm{e}$ $\mathrm{R} \$ 2.364 ”(25,5 \%)$.

\section{Instrumentos}

Os participantes responderam a um livreto contendo os instrumentos descritos no Estudo 1, a saber: o Cuestionario de Perdón en Divorcio-Separación (CPD-s; Yárnoz-Yaben \& González, 2012), versão adaptada e validada no estudo anteriormente descrito, além de um questionário sociodemográfico que permitiram caracterizar a amostra.

\section{Procedimento}

Os procedimentos adotados foram similares ao do primeiro estudo. Ressalta-se que foram seguidas todas as orientações previstas na Resolução 466/12 do Conselho Nacional de Saúde.

\section{Análises de dados}

Com o software R, efetuaram-se análises descritivas, a fim de descrever os participantes. Utilizando-se do pacote Lavaan (Rossel, 2012) executou-se uma análise fatorial confirmatória (AFC) categórica (ordinal) Weighted Least Squares Mean and Variance-Adjusted (WLSMV; Muthén \& Muthén, 2014). O wLSMV é implementado em uma matriz de correlações policóricas e considera a natureza ordinal dos dados.

Os seguintes indicadores foram utilizados para avaliar a adequação do modelo (Byrne, 2010; Tabachnick \& Fidell, 2013): (i) Comparative Fit Index (CFI) - é um índice comparativo, frequentemente, valores a partir de 0,90 são referências de um modelo ajustado; (ii) Tucker-Lewis Index (TLI) - apresenta uma medida de parcimônia entre os índices do modelo proposto e do modelo nulo, varia de zero a um, com valores acima de 0,90 como aceitáveis; (iii) Root-Mean-Square Error of Approximation (RMSEA) e seu intervalo de confiança de $90 \%$ (IC90\%), recomendando-se valores 
entre 0,05 e 0,08 , admitindo-se até 0,$10 ;$ (4) o Pclose é um indicador mais criterioso, testando a hipótese nula de RMSEA $<0,05$, deste modo, recomenda-se o Pclose $>0,05$ como indicativo de modelo ajustado.

O pacote semTools (semTools Contributors, 2016) foi adotado para calcular a confiabilidade e invariância fatorial, entre sexo (homem e mulher) e novo relacionamento (estar ou não), adotando o mesmo estimador robusto para medidas ordinais. Especificamente foram testados modelos utilizados de modo hierárquico (Cheung \& Rensvold, 2002): (i) configural, em que se verifica se o modelo fatorial (unidimensional) é igual para os grupos; (ii) métrico, em que se restringe, além da estrutura, as cargas fatoriais $(\lambda)$ e (iii) escalar adiciona a igualdade de interceptos (thresholds). Como critério de decisão para se atestar invariância foram adotados os $\Delta \chi^{2}(\mathrm{gl})$ e $\Delta \mathrm{CFI}$, sendo que o primeiro não deve ser estatisticamente significativo para ser invariante, enquanto que o segundo não deve ser igual ou superior a 0,01 (Cheung \& Rensvold, 2002; Damásio, 2013; Milfont \& Fischer, 2010).

\section{Resultados}

Tendo em conta a estrutura unifatorial original e encontrada no Estudo 1, testou-se, através da $\mathrm{AFC}$, o modelo unidimensional do CPD-S, composto por seus 5 itens. Esses itens foram então avaliados mediante a análise fatorial confirmatória categórica WLSMV a fim de conhecer o ajuste dessa estrutura fatorial aos dados empíricos. O modelo apresentou qualidade de ajuste aceitáveis, $\mathrm{CFI}=0,98, \mathrm{TLI}=$ 0,95, RMSEA $=0,07($ IC $90 \%=0,00-0,14)$ e Pclo$s e=0,19$. Os itens apresentaram cargas fatoriais $(\lambda)$ variando de 0,26 (item 2. Não posso evitar de culpar meu/minha ex-parceiro por ter causado o fim do nosso relacionamento) a 0,78 (Item 4. Apesar do comportamento do meu/minha ex-parceiro(a) ter me machucado, não guardo rancor dele). Ressalta-se que todos os pesos fatoriais $($ Lambdas $-\lambda)$ foram positivos e diferentes de zero $(\lambda \neq 0 ; z>1,96, p<0,001)$, indicando que o fator prediz satisfatoriamente as respostas aos itens. A precisão da medida foi avaliada pelo Alfa de Cronbach (correlações policóricas) e ômega de McDonald, consideradas como meritórias, 0,73 e 0,75 respectivamente. Portanto, tais resultados, reforçam as evidencias de validade e consistência interna adequadas encontradas no Estudo 1.

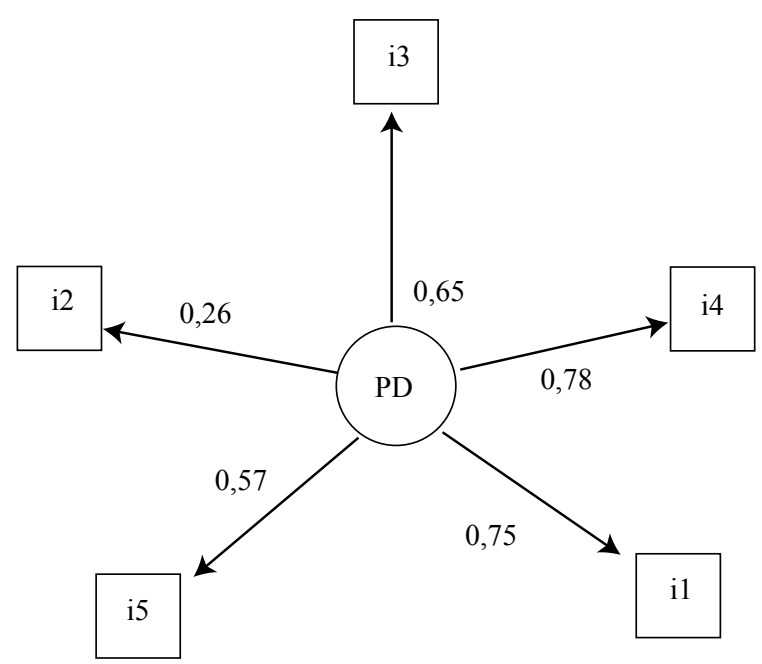

Figura 1. Estrutura Fatorial do CPD-S

Adicionalmente, foram realizadas Análises Fatoriais Confirmatórias Multi-Grupos (AFCMG) para se testar invariância da medida para as variáveis sexo e novo relacionamento, os resultados podem ser observados na tabela 2 .

Observa-se, na tabela 2, os dois grupos (sexo do participante e estar ou não em novo relacionamento) para os quais foram testadas invariâncias fatoriais, através de três modelos de restrições. Sendo plausível assumir a CPD-S invariante $(\triangle \mathrm{CFI}$ $<0,01$ ) tanto para homens e mulheres, como para quem assumiu (ou não) um novo relacionamento após o divórcio.

\section{Discussão geral}

Este artigo objetivou reunir evidências de adequação psicométrica do CPD-S, considerando sua 
Tabela 2

Invariância fatorial da CPD-S para sexo e novo relacionamento.

\begin{tabular}{|c|c|c|c|c|c|c|c|}
\hline \multicolumn{7}{|c|}{ INVARIÂNCIAS } & \multirow[t]{2}{*}{ Decisão } \\
\hline & Restrições & $\chi^{2}(\mathrm{gl})$ & cfi & RMSEA & $\Delta \chi^{2}(\mathrm{gl})$ & $\Delta \mathrm{CFI}$ & \\
\hline \multirow{3}{*}{ 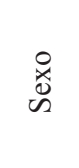 } & Configural & $29,292(10)$ & 0,949 & 0,195 & - & - & Aceitar \\
\hline & Métrica & $38,944(14)$ & 0,947 & 0,167 & $9,652(4)$ & 0,002 & Aceitar \\
\hline & Escalar & $51,090(28)$ & 0,940 & 0,126 & $12,146(14)$ & 0,007 & Aceitar \\
\hline \multirow{3}{*}{ 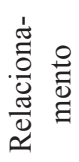 } & Configural & $34,576(10)$ & 0,943 & 0,205 & - & - & Aceitar \\
\hline & Métrica & $36,544(14)$ & 0,950 & 0,162 & $1,968(4)$ & 0,007 & Aceitar \\
\hline & Escalar & $49,582(28)$ & 0,944 & 0,121 & $13,038(14)$ & 0,006 & Aceitar \\
\hline
\end{tabular}

Nota: $* p \leq 0,05$.

validade fatorial (exploratória e confirmatória) e consistência interna (alfa de Cronbach e ômega de McDonald). Confia-se que este objetivo tenha sido alcançado, descobrindo assim, a conexão causal entre fatores latentes hipotéticos e um conjunto de itens ou indicadores, por considerá-la um dos propósitos dos modelos de análise fatorial (Markus \& Borsboom, 2013). No que se refere ao Estudo 1 , reuniram-se evidências de validade fatorial, com todas as cargas fatoriais saturando acima do ponto de corte recomendado pela literatura $(|0,30|$, Pasquali, 2010), e consistência interna do QPD-S.

No que diz respeito ao Estudo 2, a análise fatorial confirmatória, considerando a estrutura de um único fator, apresentou indicadores de ajuste satisfatórios de acordo com a literatura (e.g., $C F I$ e $T L I>0,90$ e $R M S E A<0,08$; Tabachnick \& Fidell, 2013), acontecendo o mesmo com os seus índices de consistência interna (Cohen, Swerdlik \& Sturman, 2014; Nunnally, 1978). Os achados corroboram a estrutura unidimensional como teoricamente esperada (Yárnoz-Yaben \& González, 2012).

Ademais, buscou-se avaliar a invariância dos parâmetros dos itens ao longo de grupos (sexo e está/não está em um relacionamento) com o objetivo de constatar que a estrutura fatorial, as cargas fatoriais e interceptos dos itens poderiam ser equivalentes para ambos os grupos considerados. Concomitante ao que era esperado, os resultados apontam que o CPD-S pode ser indistintamente utilizado tanto para homens e mulheres, quanto para pessoas que após o divórcio estão solteiras e que já estão em outro relacionamento, reforçando as evidências de validade e destacando as ausências de vieses de respostas. Diante disso, o CPD-S parece estar apto para avaliar diferenças reais entre tais grupos em posteriores estudos, que tenham como objetivo conhecer as magnitudes dos níveis do traço latente dos participantes (Cheung \& Rensvold, 2002; Damásio, 2013).

Ressalta-se que nesses estudos, foram feitos planejamentos de modo a utilizar análises estatísticas adequadas à natureza ordinal dos dados. Utilizando-se dos estimadores Unweighted Least Squares (ULS ou Quadrados Mínimos não-Ponderados) e Weighted Least Squares Mean- and Variance-adjusted (WLSMV ou Quadrados Mínimos Ponderados Robustos) implementados com correlações policóricas, por ser considerados recomendados e consistentes em uma situação de pesquisa que visa à relação linear entre variáveis (Asún, Rdz-Navarro \& Alvarado, 2016; Holgado-Tello et al., 2010).

Acredita-se que, nesta oportunidade, apresente uma valiosa contribuição para a produção científica crescente acerca do perdão. Proporcionando um instrumento com suas qualidades asseguradas, que torna-se alternativa, em português brasileiro para 
pesquisadores interessados em avaliar e conhecer os correlatos do perdão no âmbito do divórcio, com a vantagem de abordar os aspectos teóricos do perdão de forma breve (McCullough \& Witvliet, 2002). Considerando que no processo de adaptação e mudanças pós-divórcio, o perdão é abordado como um integrante relevante e indispensável a ser estudado, por apresentar relação positiva com o bem-estar físico e psicológico da pessoa que perdoa (Leime et al., 2012). Ademais, atitudes positivas promoverem condições para o desenvolvimento pleno e saudável dos aspectos biológicos e sociais dos seres humanos (Paludo \& Koller, 2007).

No entanto, este estudo não está isento de limitações. Destaca-se o viés amostral, por se tratar de amostras acidentais (por conveniência; Cozby, 2003), implica em avaliar os resultados sem a possibilidade de generalizá-los. Contudo, ressalta-se que não se buscou atingir a esse objetivo. Outra limitação é a ausência de uma avaliação das evidências externas do CPD-S. Nesta oportunidade são proporcionadas evidências de validade interna, porém, carece de evidências de validade externa com base na relação com outras medidas de avaliem o perdão interpessoal ou a atitude frente ao perdão durante o divórcio, além de relações com outras variáveis (e.g. adaptação ao divórcio, bem-estar subjetivo, raiva, vingança). Sendo necessário, também, em estudos futuros, buscar a associação com desejabilidade social, um fator de distorção de respostas ou promoção pessoal (Gouveia, Guerra, Souza, Santos \& Costa, 2009), e empreender esforços para compor amostras mais heterogêneas, de diferentes regiões do Brasil, replicando e refinando o uso do CPD-S.

\section{Referências}

Alves, A. P., Cúnico, S. D., Arpini, D. M., Smaniotto, A. C., \& Bopp, M. E. T. (2014). Mediação familiar: possibilitando diálogos acerca da guarda compartilhada. Pesquisas e Práticas Psicossociais, 9(2), 193-200. Recuperado de http://www.ufsj.edu.br/portal2-repositorio/File/ revistalapip/6\%20-\%20Art_\%20589\%20-\%20 Pronto(1).pdf

Asún, R. A., Rdz-Navarro, K., \& Alvarado, J. M. (2016). Developing multidimensional likert scales using item factor analysis: The case of four-point items. Sociological Methods \& Research. 45(1), 109-133. doi:10.1177/0049124114566716

Beltrão, A. G. (2012). Internalização das motivações para o perdão e seus efeitos sobre o significado de vida. (Dissertação de Mestrado. Núcleo de Psicologia Clínica Dinâmica, Universidade de Lisboa, Faculdade de Psicologia, Lisboa).

Blocher, W. G., \& Wade, N. G. (2010). Sustained effectiveness of two brief group interventions: Comparing an explicit forgiveness-promoting treatment with a process-oriented treatment. Journal of Mental Health Counseling, 32(1), 58-74. doi: 10.17744/mehc.32.1.a123852367h38057

Byrne, B. M. (2010). Structural equation modeling with Amos: Basic concepts, applications, and programmimg (2 ed.). New York, NY: Routledge.

Cheung, G. W., \& Rensvold, R. B. (2002). Evaluating goodness-of-fit indexes for testing measurement invariance. Structural Equation Modeling, 9(2), 233-255. Recuperado de http://dns2.asia.edu. tw/ ysho/YSHO-English/1000\%20China $\% 20$ (Independent)/PDF/Str\%20Equ\%20Mod9,\%20 233.pdf

Cohen, R. J., Swerdlik, M. E., \& Sturman, E. D. (2014). Testagem e Avaliação Psicológica: Introdução a Testes e Medidas (8. ${ }^{\circ}$ ed). São Paulo: $\mathrm{AMGH}$

Cozby, P. C. (2003). Métodos de pesquisa em ciências do comportamento. São Paulo: Atlas.

Dámasio, B. F. (2013). Contribuições da Análise Fatorial Confirmatória Multigrupo (AFCMG) na avaliação de invariância de instrumentos 
psicométricos. Psico-USF, 18(2), 211-220. Recuperado de http://www.scielo.br/pdf/pusf/ v18n2/v18n2a05.pdf

DeShea L. A. (2003). Scenario-based scale of willingness to forgive. Individual Differences Research, 1(3), 201-17. Recuperado de http:// psycnet.apa.org/psycinfo/2004-15380-004

DiBlasio, F. A. (1998). The use of decision-based forgiveness intervention within intergenerational family therapy. Journal of Family Therapy, 20(1), 77-96. doi: 10.1111/1467-6427.00069

Enright, R. D., \& Coyle, C. T. (1998). Researching the process model of forgiveness within psychological interventions. In E. L. Worthington (Ed.), Dimensions of forgiveness: Psychological research and theological perspectives (pp. 139161). Philadelphia: Templeton Press.

Enright, R. D., Freedman, S., \& Rique, J. (1998). The psychology of interpersonal forgiveness. In. R. D. Enright \& J. E. North (Eds.), Exploring forgiveness (pp. 46-62). London: Wisconsin Press.

Enright, R. D., \& The Human Development Study Group (1991). The moral development of forgiveness. In W. Kurtines \& J. Gewirtz (Eds.), Handbook of moral behavior and development (pp. 123-152). Hillsdale: Erlbaum.

Freedman, S., \& Knupp, A. (2003). The impact of forgiveness on adolescent adjustment to parental divorce. Journal of Divorce \& Remarriage, 39(1), 135-165. doi: 10.1300/J087v39n01_08

Gouveia, V. V., Freires, L. A., Gouveia, R. S. V., Souza Filho, J. F. D., Souza, R. V. L. D., \& Monteiro, R. P. (2015). Escala de Disposição para Perdoar: estrutura, consistência interna e invariância fatorial. Estudos de Psicologia (Campinas), 32(2), 151-161. doi: 10.1590/0103166X2015000200001

Gouveia, V. V., Guerra, V. M., Sousa, D. M. F. D., Santos, W. S., \& Costa, J. D. M. (2009). Escala de Desejabilidade Social de Marlowe-Crowne: Evidências de sua validade fatorial e consistência interna. Avaliação Psicológica, 8(1), 87-98.
Recuperado de http://pepsic.bvsalud.org/pdf/ avp/v8n1/v8n1a08.pdf

Holgado-Tello, F. P., Chacón-Moscoso, S., Barbero-García, I., \& Vila-Abad, E. (2010). Polychoric versus Pearson correlations in exploratory and confirmatory factor analysis of ordinal variables. Quality \& Quantity, 44(1), 153-166. doi:10.1007/s11135-008-9190-y

Lamela D., Figueiredo B., Bastos A., \& Martins H. (2014). Psychometric properties of the Portuguese version of the Posttraumatic Growth Inventory short form among divorced adults. European Journal of Psychological Assessment, 30(1), 3-14. Doi: 10.1027/1015-5759/a000161.

Lara, S. A. D. (2014). ¿Matrices Policóricas/Tetracóricas o Matrices Pearson? Un tudio metodológico. Revista Argentina de Ciencias del Comportamiento, 6(1), 39-48. Recuperado de http:// www.redalyc.org/articulo.oa?id=333430869006

Leime, J., Luna, V., Leite, J., \& Neto, J. R. (2012). O Pensamento do Perdão em Idosos Institucionalizados. Psico, 43(1), 69-76. Recuperado de http://revistaseletronicas.pucrs.br/ojs/index. php/revistapsico/article/view/11100/7620

Lorenzo-Seva, U., \& Ferrando, P. J. (2013). FACTOR 9.2: A Comprehensive Program for Fitting Exploratory and Semiconfirmatory Factor Analysis and IRT Models. Applied Psychological Measurement, 37(6), 497-498. doi:10.1177/0146621613487794

Lorenzo-Seva, U., Timmerman, M. E., \& Kiers, H. A. L. (2011). The Hull Method for Selecting the Number of Common Factors. Multivariate Behavioral Research, 46(2), 340-364. doi:10. 1080/00273171.2011.564527

Markus, K. A., \& Borsboom, D. (2013). Frontiers of test validity theory: Measurement, causation, and meaning (Multivariate Applications Series). New York: Routledge.

Mazor, A., Batiste-Harel, P., \& Gampel, Y. (1998). Divorcing spouse's copping patterns, attachment bonding and forgiveness processes in the post-divorce experience. Journal of Divorce 
\& Remarriage, 29(1), 65-81. doi: 10.1300/ J087v29n03_04

McCullough, M. E. (2000). Forgiveness as human strength: Theory, measurement, and links to well-being. Journal of Social and Clinical Psychology, 19(1), 43-55. doi: 10.1521/jscp.2000.19.1.43

McCullough, M. E., Root, L. M., \& Cohen, A. D. (2006). Writing about the personal benefits of a transgression facilitates forgiveness. Journal of Consulting and Clinical Psychology, 74(5), 887-897. doi: 10.1037/0022-006X.74.5.887

McCullough, M. E., \& Witvliet, C. (2002). The psychology of forgiveness. In C. R. Snyder, \& S. Lopez (Eds.), Handbook of Positive Psychology (pp. 446-458). NewYork: Oxford University Press.

Milfont, T. L., \& Fischer, R. (2010). Testing measurement invariance across groups: Applications in cross-cultural research. International Journal of Psychological Research, 3(1), 2011- 2084. Recuperado de https://dialnet.unirioja.es/descarga/articulo/3296462.pdf

Muthén, L. K., \& Muthén, B. O. (2014). Mplus user's guide. (Seventh Ed.). Los Angeles: Muthén \& Muthén.

Neto, F. M., \& Mullet, E. (2012). Perdão e reconciliação entre grupos: a perspectiva timorense. Ariús, 18(1), 31- 54. Recuperado de http://www. ch.ufcg.edu.br/arius/01_revistas/v18n2/00 arius_v18_n2_2012_edicao_completa.pdf

Nunnally, J. C. (1978). Psychometric theory. New York: McGraw-Hill Inc.

Paludo, S. S., \& Koller, S. H. (2007). Psicologia positiva: uma nova abordagem para antigas questões. Paidéia, 17(36), 9-20. Recuperado de http://www.scielo.br/pdf/paideia/v17n36/ v17n36a02.pdf

Pasquali, L. (2010). Instrumentação psicológica: Fundamentos e práticas. Porto Alegre, RS: Artmed.

R Core Team (2015). R: A language and environment for statistical computing. R Foundation for Statistical Computing, Vienna, Austria. Recuperado de http://www.R-project.org/.

Rique, J., Camino, C. P. S., Santos, W. S., \& Gouveia, V. V. (2009). Análise Confirmatória da Escala de Atitudes para o Perdão - EFI. Avaliação Psicológica, 8(2), 169-178. Recuperado de http:// pepsic.bvsalud.org/pdf/avp/v8n2/v8n2a03.pdf Rizopoulos, D. (2006). ltm: An R package for Latent Variable Modelling and Item Response Theory Analyses. Journal of Statistical Software, 17(5), 1-25. Recuperado de http://www.jstatsoft.org/ v17/i05/

Rossel, Y. (2012). Lavaan: na R package for structural equation modeling. Jounal of Statistical Sofware, 48(2), 1-36. doi: 10.18637/jss.v048.i02 Rye, M. S., Folck, C. D., Heim, T. A., Olszewski, B. T., \& Traina, E. (2004). Forgiveness of an ex-spouse: How does it relate to mental health following a divorce? Journal of Divorce and Remarriage, 41(3), 31-51. doi: 10.1300/ $\mathrm{J} 087 \mathrm{v} 41 \mathrm{n} 03$ 02

Rye, M. S., Loiacono, D. M., Folck, C. D., Olszewski, B. T., Heim T.A., \& Madia, B. P. (2001). Evaluation of the psychometric properties of two forgiveness scales. Current Psychology: Developmental, Learning, Personality, Social, 20(3). 260-277. Recuperado de http://pages. uoregon.edu/bolszews/CPforgiveness.pdf

Samejima, F. (1969). Estimation of a latent ability using a response pattern of graded score. $P s i$ chometrika Monograph, 34(17). Recuperado de https://www.psychometricsociety.org/sites/ default/files/pdf/MN17.pdf

SemTools Contributors. (2016). semTools: Useful tools for structural equation modeling. $R$ package version 0.4-12. Recuperado de http:// cran.r-project.org/package $=$ semTools

Strelan, P., \& Covic, T. (2006). A review of forgiveness process models and a coping framework to guide future research. Journal of Social and Clinical Psychology, 25(10), 1059-1085. doi: 25:1059-1085. 
Subkoviak, M. J., Enright, R. D., Wu, C. R., Gassin, E. A., Freedman, S., Olson, L. M., \& Sarinopoulos, I. (1995). Measuring interpersonal forgiveness in late adolescence and middle adulthood. J Adolesc, 18(6), 641-55. doi:10.1006/ jado.1995.1045

Tabachnick, B. G., \& Fidell, L. S. (2013). Using multivariate statistics (6. ${ }^{\mathrm{a}}$ ed.). Nova Iorque: Allyn \& Bacon

Toussaint, L. L., Owen, A. D., \& Cheadle, A. (2012). Forgive to live: forgiveness, health, and longevity. Journal of Behavioral Medicine, 35(4), 375-386. Doi: 10.1007/s10865-011- 9362-4

Wade, S. H. (1989). The development of a scale to measure forgiveness. (Tese de doutorado. Fuller Graduate School of Psychology, Passadena, CA).

Worthington, E. L. (2005). Handbook offorgiveness. New York: Routledge.

Recebido:Julho 18, 2016

Aprovado: Dezembro 12, 2016
Yárnoz-Yaben, S., Comino, P., \& Garmendia, A. (2012). Ajuste al divorcio de los progenitores y problemas de conducta en hijos de familias separadas. Infancia y rendizaje, 35(1), 35-48. doi: 10.1174/021037012798977485

Yárnoz-Yaben, S., Garmendia, A., \& Comino, P. (2015). Looking at the Bright Side: Forgiveness and Subjective Well-Being in Divorced Spanish Parents. Journal Happines Studies, 17, 1905-1919.doi: 10.1007/s10902-015-9677-x

Yárnoz-Yaben, S., \& González, C, P. (2012). Un instrumento para la evaluacio'n del perdo'n en el a'mbito del divorcio y la separacion. International Journal of Psychology and Psychological Therapy, 12 (1), 49-58. Recuperado de http:// www.redalyc.org/articulo.oa? id=56023335004 